\title{
ARTICLE
}

\section{In vivo imaging reveals unique neutrophil transendothelial migration patterns in inflamed intestines}

\author{
David P. Sullivan ${ }^{1}$, Triet Bui ${ }^{1}$, William A. Muller ${ }^{1}$, Veronika Butin-Israeli ${ }^{1}$ and Ronen Sumagin ${ }^{1}$
}

\begin{abstract}
Neutrophil (PMN) infiltration of the intestinal mucosa is a hallmark of gastrointestinal inflammation, with significant implications for host defense, injury and repair. However, phenotypic and mechanistic aspects of PMN recruitment in inflamed intestines have not been explored in vivo. Using novel epithelial/PMN fluorescence reporter mice, advanced intravital imaging and 3D reconstruction analysis, we mapped the microvasculature architecture across the intestinal layers and determined that in response to Salmonella/ endotoxin-induced inflammation, PMN transendothelial migration (TEM) was restricted to submucosal vessels. PMN TEM was not observed in villus or crypt vessels, proximal to the epithelium that underlies the intestinal lumen, and was partially dependent on (C-X-C motif) ligands 1 (CXCL1) and 2 (CXCL2) expression, which was found to be elevated in the submucosa layer. Restricted PMN extravasation at the submucosa and subsequent PMN interstitial migration may serve as a novel regulatory step of PMN effector function and recruitment to the luminal space in inflamed intestines.
\end{abstract}

Mucosal Immunology (2018) 11:1571-1581; https://doi.org/10.1038/s41385-018-0069-5

\section{INTRODUCTION}

Polymorphonuclear leukocytes (PMNs) can function as a doubleedged sword promoting tissue injury and contributing to reestablishment of tissue homeostasis. ${ }^{1,2}$ As such, en masse PMN infiltration of mucosal surfaces including respiratory system, urinary and the gastrointestinal tracts is often associated with disruption of the critical barrier function and tissue injury. ${ }^{3,4}$ However, emerging evidence also convincingly implicates PMNs in the resolution of inflammation and wound repair. ${ }^{5-7}$

PMN accumulation in the mucosa and luminal spaces is a hallmark of inflammatory bowel disease (IBD), a debilitating disorder affecting over a million individuals in the United States alone. ${ }^{8}$ Although PMN presence in the intestinal tissue is generally viewed as detrimental and often correlates with disease symptoms, pro-repair functions of PMNs in the intestinal lumen are increasingly recognized. For example, PMN binding to and ligation of luminal receptors, such as intercellular adhesion molecule-1 (ICAM-1), has been suggested to promote epithelial proliferation and wound repair. ${ }^{9}$ As with IBD, infection by common enteric pathogens, including Salmonella or Listeria, leading to symptomatic disease, elicits PMN recruitment and accumulation in the intestinal mucosa and the lumen. ${ }^{10}$ Here too, while critical for host defense, excessive PMN tissue accumulation contributes to tissue dysfunction. ${ }^{3}$ Given these important implications of PMN trafficking in the mucosa, there has been a long-standing interest in understanding the molecular basis and functional consequences of PMN interaction with the endothelium as they cross the vascular wall, and subsequently with the epithelial layer on their way to the intestinal lumen.

Many in vitro and in vivo models have been employed to study PMN transendothelial migration (TEM) and help define critical steps and key molecules involved in this process. However, only the use of emerging, advanced intravital imaging techniques allowed visualization and detailed examination of PMN interactions with endothelial cells (ECs) in various vascular beds and disease models. ${ }^{11}$ Combined, these studies revealed that PMN activation, ${ }^{12}$ endothelial adhesion molecule density, ${ }^{13,14}$ fluid shear forces ${ }^{15}$ and geometry of the vascular bed itself ${ }^{16}$ can $^{16}$ significantly influence this process. Most PMN TEM in response to inflammation take place at post-capillary venules $(15-40 \mu \mathrm{m}$ in diameter). ${ }^{17}$ At these sites, PMNs undergo sequential adhesive steps that include selectin-mediated rolling interactions and integrin-dependent PMN adhesion, followed by luminal locomotion (crawling) and terminating with TEM. ${ }^{18}$ Interestingly, as was observed in lung circulation, the capillary bed can also support PMN TEM. ${ }^{16}$ In fact, in response to distal pulmonary airspace inflammation, alveolar capillaries are the principal site of leukocyte extravasation. ${ }^{19}$ Given the capillary size $(2-15 \mu \mathrm{m}$ in diameter), a simple physical trapping of PMNs rather than tethering interactions may be sufficient to initiate TEM. ${ }^{19,20}$ Importantly, across all vascular beds, including post-capillary venules and capillaries, the presence of chemotactic gradients and activation/upregulation of adhesive receptors is critical for PMN TEM. ${ }^{21}$

Although PMN infiltration of the intestinal mucosa has long been known to be a prominent histological feature of intestinal inflammation, the fundamental mechanisms underpinning PMN TEM in the gut vasculature have largely gone unexplored. Furthermore, how the complex architecture and the multilayered structure of the intestines reflect on the PMN extravasation patterns is not clear. This work details the use of an advanced intravital imaging setup and a three-dimensional (3D) reconstruction analysis to map the microvasculature architecture across the layers of the small intestine and determine the patterns of PMN extravasation and recruitment to inflamed epithelium. As new information continues to emerge regarding the expanded life span of PMNs in tissue and the heterogeneity of PMN subsets and

'Department of Pathology, Feinberg School of Medicine, Northwestern University, Chicago, IL, USA

Correspondence: Ronen Sumagin (ronen.sumagin@northwestern.edu)

Received: 12 March 2018 Revised: 28 June 2018 Accepted: 28 June 2018

Published online: 13 August 2018 

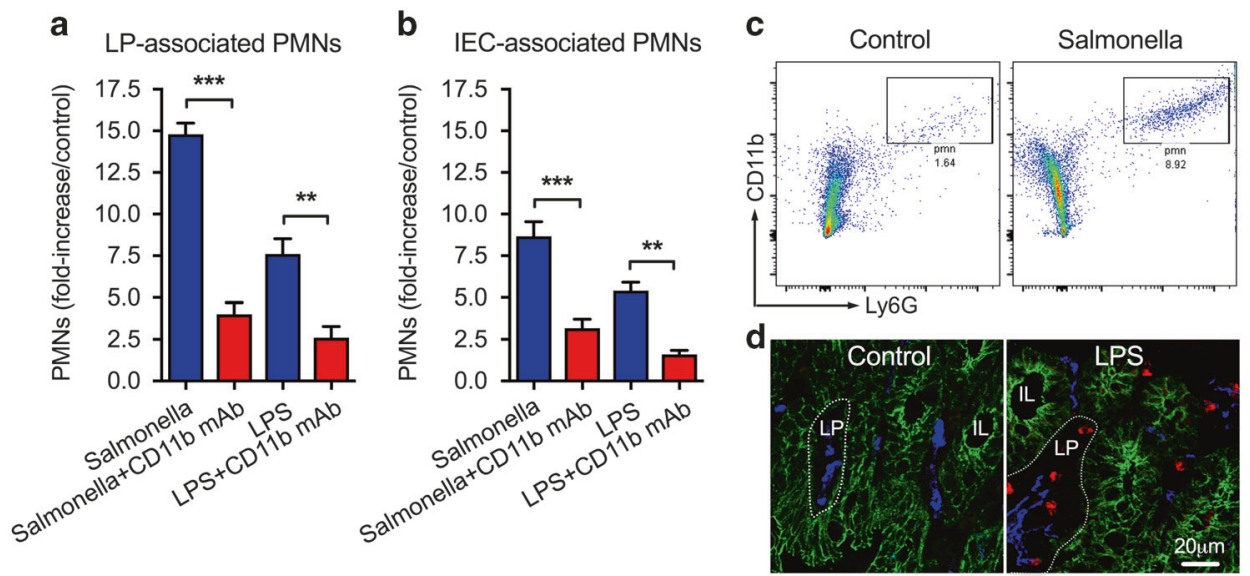

Fig. 1 Pathogenic stimulus triggers PMN infiltration of the intestinal mucosa. Intestinal inflammation was induced by either Salmonella or LPS treatment. Following treatment, surface epithelia with the associated PMNs were separated from lamia propria cells by tissue digestion. a Flow cytometry was performed to quantify the number of PMNs recruited to the lamina propria (LP). PMNs were identified by serially gating on CD45- and CD11b/Ly6G-positive cells. b Similar flow cytometry analysis was performed on the IEC fraction. c Representative flow diagrams show increased PMN fraction in LP-cell isolate. d Representative immunofluorescence images, taken from cryosections of control and LPSinflamed intestinal tissue, showing increased PMN (stained with anti-Ly6G, red) presence in inflamed intestinal mucosa. Vessels (blue) and epithelial cells (green) were stained using anti-PECAM-1 and E-cadherin antibodies, respectively. IL intestinal lumen. Data shown for (a, b) are an average of at least three independent experiments for each condition, ${ }^{* *} p<0.01{ }^{* * *} p<0.001$. Diagrams for (c, d) are representative of three independent experiments

function, our understanding of the roles of PMNs in inflammation and pathology is rapidly evolving. As such, the specific activation state of the PMN and the immediate microenvironment and milieu of the intestinal regions likely plays a critical role in regulating TEM and the key effector functions of PMNs.

\section{RESULTS}

Pathogenic stimulus triggers PMN infiltration of the intestinal mucosa

In the setting of acute intestinal inflammation triggered by invading pathogens, such as Salmonella, PMNs are recruited to the intestinal mucosa and the epithelial layer. ${ }^{10,22}$ To examine this process in more detail, intestinal inflammation was induced by either oral gavage of Salmonella $\left(10^{8}\right.$ in $100 \mu$ l by gavage, $24 \mathrm{~h}$ before sample collection) or by intraluminal administration of bacterial endotoxin (lipopolysaccharide (LPS), $100 \mu \mathrm{g} / \mathrm{ml}$ in $200 \mu \mathrm{l}$, $2 \mathrm{~h}$ before sample collection). In both inflammation models, PMN recruitment to intestinal epithelial cells (IECs) and the deeper lamina propria mucosa was quantified. In these experiments, intestines were harvested, cleaned and subjected to brief mechanical stress (in DTT/EDTA solution), which separates intestinal epithelium from the rest of the mucosa and the underlying muscular layer. ${ }^{23-25}$ Both resulting fractions were processed to produce single-cell suspensions, which were then analyzed by flow cytometry to determine recruitment of PMNs. As expected, Salmonella treatment triggered robust PMN extravasation from the blood vessels and accumulation within the deeper layers of the lamina propria (LP) and the epithelium (Fig. 1a, b and representative flow micrograph, Fig. 1c). Importantly, although to a slightly lesser degree, administration of LPS into the intestinal lumen (high dose of $100 \mu \mathrm{g}$ ) recapitulated a similarly robust but regulated PMN response (Fig. $1 \mathrm{a}, \mathrm{b}$ and representative immunofluorescence images, Fig. 1d). Inhibition of PMN interactions with the vascular wall by intravenous (i.v.) administration of functionblocking $\mathrm{CD} 11 \mathrm{~b}$ antibody $(\mathrm{Ab})$ significantly decreased the number of tissue PMNs in both models of intestinal inflammation (Fig. 1a, b). These data suggest that PMN recruitment induced by both Salmonella and LPS follows well-established leukocyte-EC adhesion cascade. Because LPS produced a more consistent inflammatory response, on par with that seen by others, ${ }^{26,27}$ and was significantly less challenging experimentally, LPS was used in all subsequent experiments.

Villus microcirculation does not support PMN TEM

Given the observed PMN infiltration of the intestinal epithelium in response to Salmonella infection or LPS treatment, we sought to examine the vasculature in detail to determine the extent to which these vessels could support PMN TEM. We first used intravital multiphoton microscopy and epithelial/innate immune cell reporter mice (E-cadherin CFP/LysM-eGFP, such that IECs are blue, PMNs eGFP ${ }^{\text {hi }}$ and macrophages eGFPlow) to examine the villi from the side of the intestinal lumen, using experimental setup similar to that previously described. ${ }^{28,29}$ Dextran-Texas Red (70 $\mathrm{kDa}$, co-injected with LPS into the intestinal lumen) and antiPECAM-1-conjugated to Alexa Fluor ${ }^{\circledR} 555$ fluorophore (injected i.v.) were used to demarcate lumen/villus boundaries and the vasculature respectively. By opening up the ileal intestine, we were able to visualize villus architecture from the tip down to a depth of $\sim 150-200 \mathrm{~nm}$ (Fig. 2a and schematic Fig. 2b). Each villus was perfused by an extensive vascular network, with vessels (ranging from 5 to $10 \mu \mathrm{m}$ in diameter) positioned in close proximity to the epithelial lining (from the tip to the base of the villus, 3D reconstruction of crypts, Fig. $2 \mathrm{c}$ and zoom in on vasculature, Fig. 2d).

Real-time luminal imaging of the villus vasculature under control conditions revealed free-flowing PMNs, with velocities greater than $100 \mu \mathrm{m} / \mathrm{s}$ that rarely engaged in interactions with the vessel wall (Fig. 2e, g). Both Salmonella infection and LPSmediated inflammation induced PMN interactions with the vessel wall to a similar degree (shown for LPS, Fig. 2e). Under these conditions, a significant portion of the free-flowing PMNs exhibited transient attachments to the vessel wall, lasting between 1 and $5 \mathrm{~s}$ (tethering), with translational velocities of 5.3 $\pm 0.8 \mu \mathrm{m} / \mathrm{s}$ (Fig. 2f and Supplemental Video 1). PMN firm adhesion (remaining attached to the vessel wall for more than $20 \mathrm{~s}$ ) was also observed (Fig. $2 \mathrm{~g}$ and Supplemental Video 1). However, although PMN adhesion, a well-documented prerequisite step for TEM, ${ }^{30-32}$ was seen, PMN TEM was never observed in villus vasculature (despite extensive imaging analysis of more than 18 fields in 9 mice and imaging times of up to $45 \mathrm{~min}$, which is more than sufficient time for TEM to take place ${ }^{33,34}$ ). 


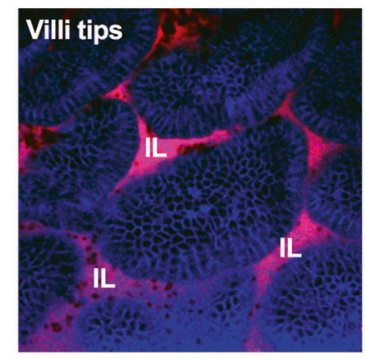

Lumen $(z=0 \mu \mathrm{m})$

b

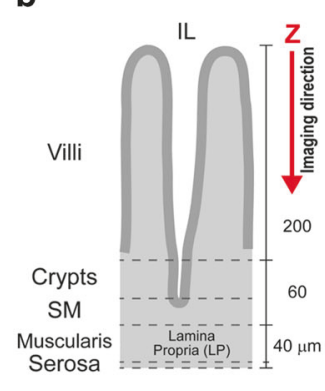

C

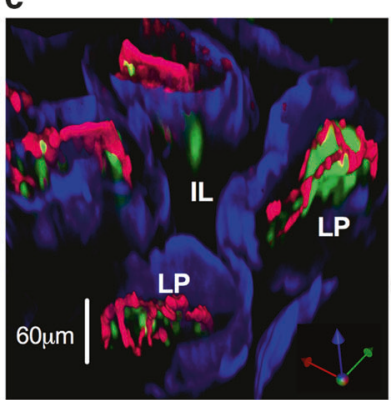

d

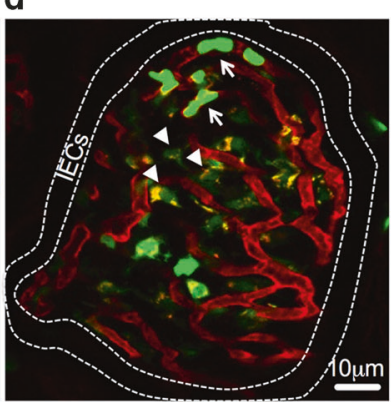

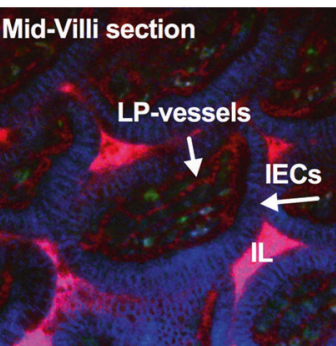

$z=100 \mu \mathrm{m}$

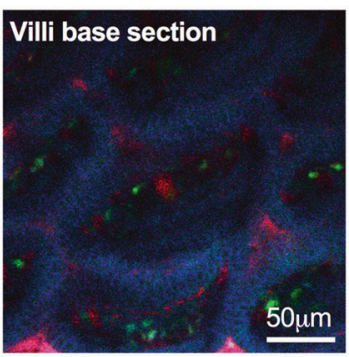

$(z=150 \mu \mathrm{m})$ c e

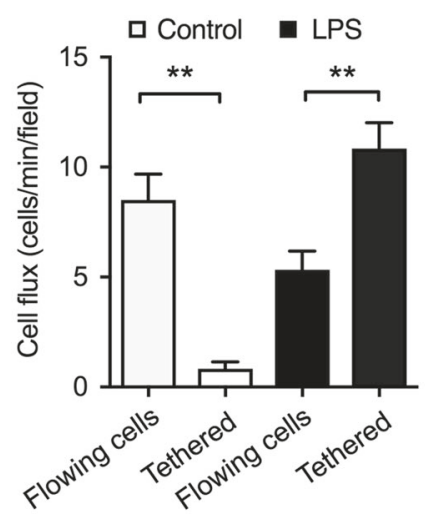

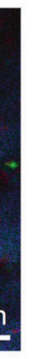




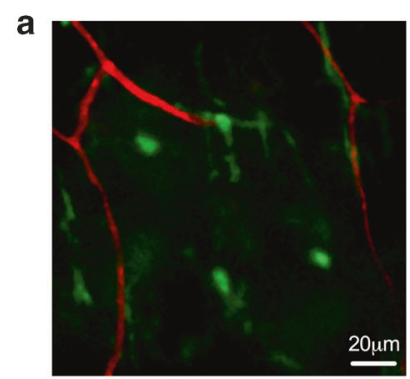

Serosa $(z=0 \mu \mathrm{m})$

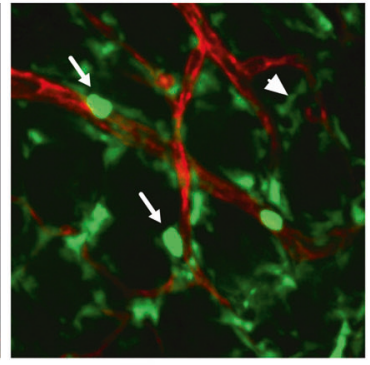

$z=80 \mu \mathrm{m}$

\section{西}
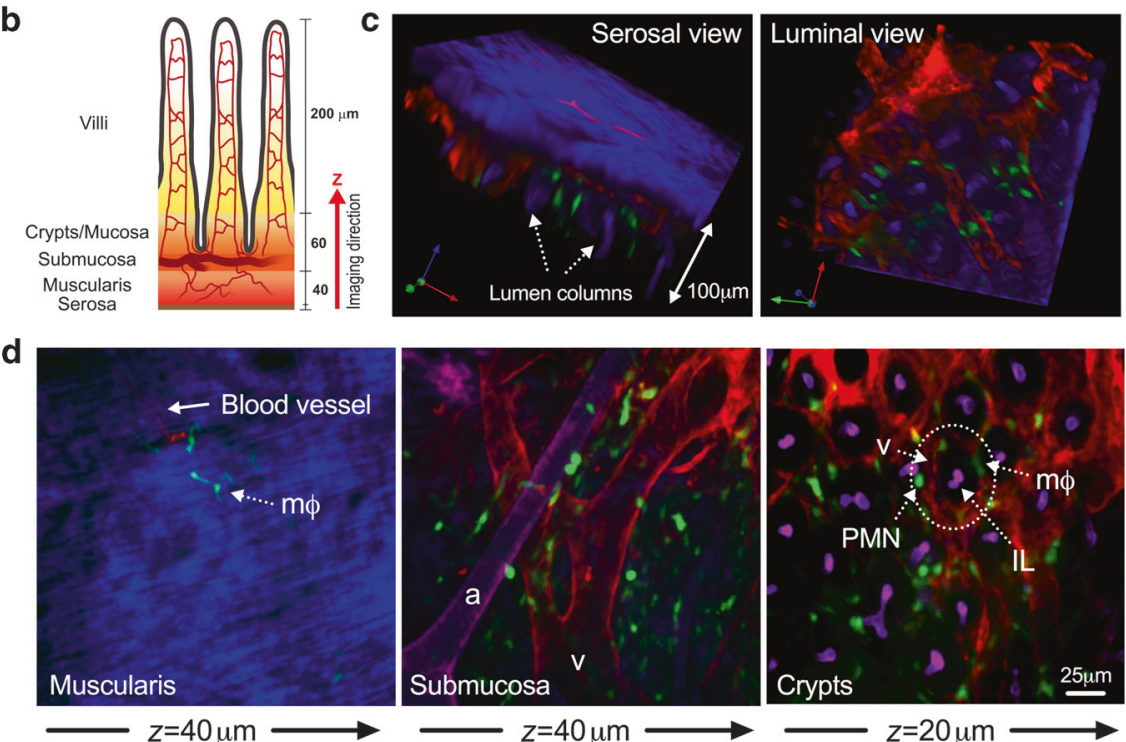

Fig. 3 Mapping of vasculature across intestinal layers by serosal imaging. a Immune cell reporter mice (LysM-eGFP) were used for serosal imaging to examine the localization of microvessels (labeled with fluorescent non-blocking anti-PECAM-1 antibodies) across intestinal layers. Arrows indicate eGFPhi PMNs, while arrowheads indicate eGFPlow tissue macrophages. b A schematic representation of the intestinal layers (including approximate length of each layer) as determined by serosal imaging. c Segments of the small intestine of LysM-GFP mice were extracted, PFA fixed, permeabilized and fluorescently stained for EC marker PECAM-1 (red) and actin (blue). A series of 120 images were acquired in Z-direction with increments of $1 \mu \mathrm{m}$. Representative 3D reconstructions show superficial vessels localized to actin-rich serosa/ muscularis layer (serosal view, left panel) and larger vessels localized to the submucosal layer (luminal view, right panel). d Representative Zprojections of the images from the indicated layers showing microvessels in the actin-rich muscularis (left panel), deeper larger vessels in the submucosa (middle panel) and crypt vasculature (right panel). Dotted circle highlights vessels encircling a crypt, at the center of which are the crypt epithelial cells that stain positive for actin; a arteriole, $v$ venule, $\mathrm{m} \Phi$ macrophage, IL intestinal lumen. All images are representative of three independent experiments

morphology of the crypt lumens, and likely represent the staining of actin in crypt epithelial cells (Fig. 3c, d). Each crypt was encircled by a network of small vessels that appeared largely sinusoidal in nature. In inflamed tissue, the interstitial space between the crypts and vessels was replete with bright eGFPhi PMNs and dim eGFPlow macrophages.

PMN TEM is restricted to the submucosa vessels

By imaging from the serosal side, we were able to resolve the precise location of neutrophils in the various layers in high detail relative to the vasculature. Compared to control, there was a substantial increase in the number of PMNs observed outside of the blood vessels in all strata of the LPS-inflamed small intestine (Fig. 4a). However, the most striking difference was observed in the submucosa layer, which had an $\sim 10$-fold increase over the control (compared to 2.5- and 3-fold increase in the inflamed muscularis and crypt layers, respectively, Fig. 4b).

Because of the relatively high number of PMNs observed in the submucosa, we next sought to determine whether submucosa vessels preferentially support PMN TEM. We performed intravital imaging from the serosal surface on control and LPS-inflamed intact intestines. With this method, we were able to stain and resolve the muscularis, submucosa and the crypt vasculature in live LysM-eGFP mice. PMNs in unstimulated muscularis and crypt vessels were observed free-flowing in the blood stream with velocities ranging between 100 and $150 \mu \mathrm{m} / \mathrm{s}$, but rarely engaging or interacting with vessel walls. Upon LPS treatment, the number of PMNs observed per field in both muscularis and crypt layers was significantly increased. Under these conditions, $>70 \%$ of all PMNs were found to transiently interact with the vessel wall (less than $1 \mathrm{~s}$ /interaction), but no continued slow rolling was seen. The average translational velocity of PMNs was significantly reduced, likely resulting from increased PMN contact with the vessel walls. Displacement trajectories of representative PMNs and their initial locations for each condition are indicated by a white dashed line and a star, respectively (Fig. 5a, e and quantified in Fig. 5b, c for muscularis, and F, G for crypt vessels). Importantly, in both strata, PMN firm adhesion was substantially increased (Fig. 5d, h). Despite the observed PMN adhesion, no PMN TEM from these vessels was observed (Supplemental Videos 2 and 3 show PMNs in muscularis and crypt vessels, respectively). As with villus vasculature, at least 9 mice/condition were used in imaging experiments to substantiate this conclusion. This suggests that the larger submucosal vessels upstream 

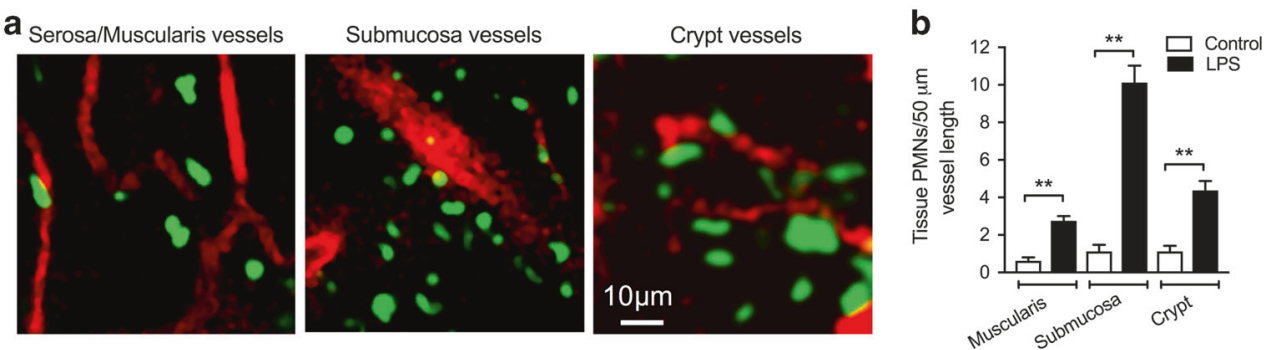

Fig. 4 PMNs infiltrate all layers of the inflamed intestine. Local inflammation was induced by intraluminal administration of LPS $(100 \mu \mathrm{g} / \mathrm{ml}, 2 \mathrm{~h}$ before imaging) in ligated intestinal loops of LysM-eGFP reporter mice. The number of tissue-infiltrated PMNs (green) in the serosa/muscularis, the submucosa and the crypt layers was quantified and compared to control conditions. a Images representative of three independent experiments showing PMN infiltration of the intestinal layers. Fluorophore-conjugated non-blocking anti-PECAM-1 antibody was injected i.v. to visualize the vessels (red). b Quantification of tissue PMNs. Data are shown as an average of three independent experiments, ${ }^{* *} p<0.01$

of crypt vasculature likely support the bulk of PMN TEM. Consistent with this idea, an increased number of rolling PMNs was seen in inflamed submucosal vessels (Fig. 5j). With LPS stimulation, PMN rolling velocities were significantly decreased $(24.2 \pm 1.3$ vs. $6.7 \pm 0.4 \mu \mathrm{m} / \mathrm{s}$, indicative of PMN activation, Fig. $5 \mathrm{k})$ and PMN adhesion was substantially increased (Fig. $5 \mathrm{I}$ and Video 4). Further imaging experiments revealed that $2-3$ PMNs $(\sim 30 \%)$ of all adhered PMNs at the vessel wall (6-9 cells/100 $\mu \mathrm{m}$ vessel length) transmigrated out of the vessel into the surrounding tissue. In these experiments, movies were acquired for $20-30 \mathrm{~min}$ and 10 fields in 5 mice were analyzed. Representative images of two PMNs extravasating from the submucosa vessel are shown in a time-lapse image sequence (Fig. $5 \mathrm{~m}$ and Video 5). To account for the possible effect of the genetic model, TEM was quantitated in heterozygous and homozygous LysM-eGFP; no difference was observed (data not shown).

CXCL1/CXCL2 and other intrinsic factors drive PMN TEM in the submucosa

$\mathrm{EC} \mathrm{Ca}^{2+}$ is a well-known, critical regulator of PMN TEM. ${ }^{37,38}$ We hypothesized that the preferential localization of PMN TEM to the submucosa vessels could be a result of enhanced $\mathrm{Ca}^{2+}$ responses in those ECs, compared to ECs in the muscularis layer or intestinal villi. To examine this possibility, we performed intravital microscopy on control and LPS-inflamed vessels in mice expressing the $\mathrm{Ca}^{2+}$ reporter GCaMP in ECs (GCaMP3 ${ }^{\text {flox/flox }}$ expression driven by the CAG promoter and restricted to ECs using VE-Cadherin-Cre). In this setup, an increase in the intracellular $\mathrm{Ca}^{2+}$ is observed directly as corresponding increase in fluorescence intensity. ${ }^{39}$ We therefore quantitated the fluorescence intensity in vessels in the indicated strata of inflamed and control intestines. In response to LPS stimulation, increased signal intensity (and thus, increased EC $\mathrm{Ca}^{2+}$ ) was observed in vessels in the submucosa, muscularis (Fig. 6a, b) and villus vasculature (not shown). However, the fold change (ratio of signal measured with LPS versus control) was not different between the two layers, suggesting that $\mathrm{Ca}^{2+}$ response was not the reason for the restricted PMN TEM in submucosal vessels.

Signaling by chemokines is another important element to regulate PMN TEM. ${ }^{40,41}$ We thus sought to test the possibility that expression of several major PMN chemokines, including (C-X-C motif) ligands 1, 2 and 5 (CXCL1, CXCL2 and CXCL5), varies in the different strata. To quantitate this, we used a previously established protocol ${ }^{42}$ to serially section control and LPSinflamed intestinal tissue. This allowed us to harvest and pool representative sections from each of the intestinal layers in question (schematic shown in Fig. 6c). These samples were then processed and analyzed by quantitative reverse transcriptionpolymerase chain reaction (qRT-PCR) and enzyme-linked immunosorbent assay (ELISA) for messenger RNA (mRNA) and protein content, respectively. The quality and purity of the separation of the strata was confirmed by histological analysis (Fig. 6d) and the differential expression of villus surface (Claudin 4, KIf4), crypt base (Claudin 2 and KIf5) and smooth muscle actin-a markers (Fig. 6e). Transcription (Fig. 6f) and protein (Fig. 6g) levels for CXCL1 and CXCL2, but not CXCL5, were found to be elevated in the submucosal/crypt layer when compared to villi, suggesting that localization of PMN TEM to the submucosa vasculature during intestinal inflammation could be due to elevated CXCL1/CXCL2 levels.

Both CXCL1 and CXCL2 have been previously shown to have high-affinity binding interactions with PMN-expressed CXC chemokine receptor 2 (CXCR2). ${ }^{43,44}$ To confirm the role of CXCL1/CXCL2 and CXCR2 binding interactions in mediating PMN TEM in the submucosa vessels, mice were treated with the function-blocking anti-CXCR2 Ab $(100 \mu \mathrm{g}$ of $\mathrm{Ab}$ in $100 \mu \mathrm{l}$, intraperitoneal (i.p.) injection) 30 min prior to LPS administration, and then quantified PMN TEM and recruitment to IECs. CXCR2 inhibition significantly reduced PMN TEM (PMNs in the submucosa layer, Fig. 6h) and reduced by $\sim 70 \%$ the LPS-induced PMN infiltration of IECs (Fig. 6i), confirming the role of PMN receptor/ ligand interactions in TEM.

Finally, we asked whether higher levels of CXCL1 and CXCL2 are sufficient to drive localized PMN TEM. In these experiments, CXCL1 or CXCL2 were administered into the lumen of ligated intestinal loops, ${ }^{26,45}$ to experimentally alter their tissue distribution in such way that the highest concentration of chemokines is now adjacent to the villus circulation. We then monitored PMN TEM and infiltration of IECs using intravital microscopy and parallel flow cytometry analyses. Although both chemokines induced PMN recruitment to the IEC layer (Fig. 6j), TEM was still restricted to the submucosa vasculature (Fig. $6 \mathrm{k}$, shown for $\mathrm{CXCL} 1$ ), as was observed with LPS.

These findings suggest that although CXCL1 and CXCL2 can drive PMN TEM, other yet undefined intrinsic differences in vascular ECs or the immediate microenvironment within the intestinal strata also regulate PMN TEM in inflamed intestines.

\section{DISCUSSION}

In the presence of inflammatory cues, PMNs are rapidly mobilized from the vasculature to surrounding tissue. Although PMN infiltration of the intestinal mucosa and the luminal space is a prominent feature of IBD and enteropathogenic infections, ${ }^{2,22}$ to our knowledge, only one prior study attempted to study PMN recruitment in inflamed intestines by using live imaging approaches. $^{27}$ This work determined the extracellular signalregulated kinase activity to be crucial in PMN trafficking across intestinal tissue. The stratification of the intestinal wall has long been known to be important for the overall function of the organ. Here we sought to investigate whether the various strata also 

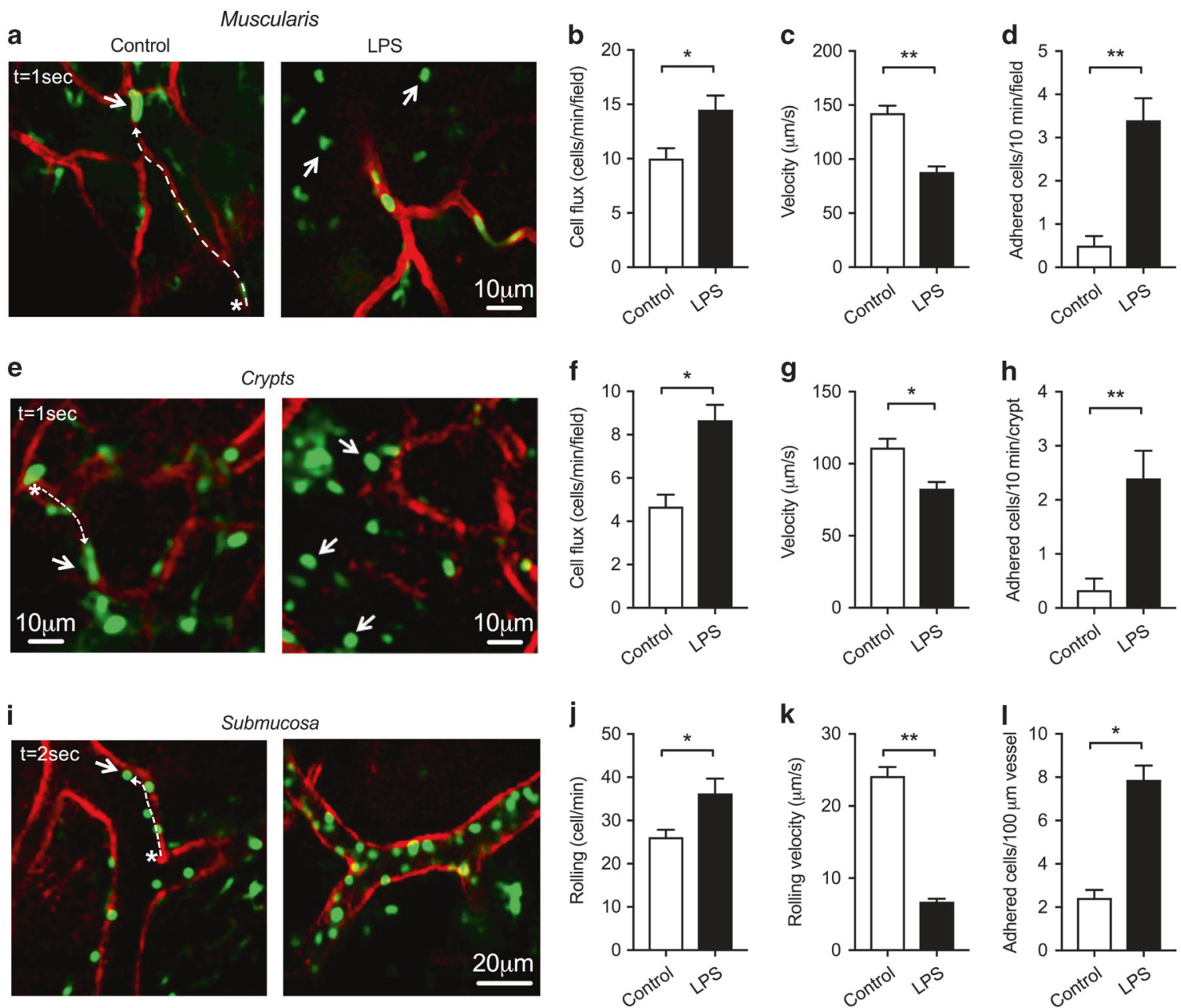

m

Time-lapse, PMN TEM in submucosa vessel
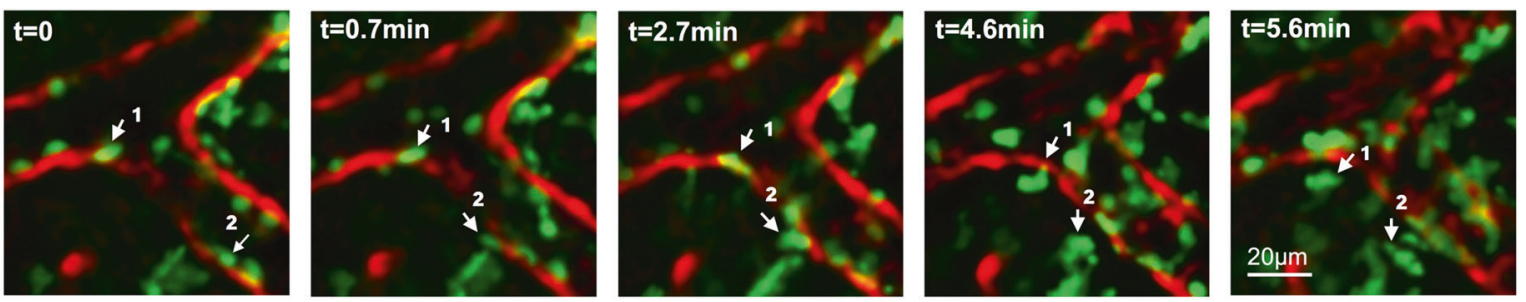

Fig. 5 PMN TEM is restricted to the submucosa vessels. Immune cell reporter mice (LysM-eGFP) were used for imaging from the serosal side. Vasculature was outlined using fluorescent non-blocking anti-PECAM-1 (red). PMN behaviors, including cell fluxes (visualized PMNs per minute per field), velocity and adhesion (PMN attachment to the vessel wall for longer than $20 \mathrm{~s}$ ), were analyzed in control and LPS treated (a-d) muscularis, (e-h) crypts and (i-I) submucosa by real-time image acquisition and off-line analysis using ImageJ. For control images (left panels) the displacement trajectory of a representative PMN (over 1-2 s, as indicated in the left corner) is indicated by the white dashed line. The PMN initial and final position is indicated by the star and an arrow, respectively. For inflamed condition (right panel), tissue PMNs are shown by the white arrows. All images are representative of three independent experiments. Data are shown as an average of five independent experiments, ${ }^{*} p<0.05,{ }^{* *} p<0.01$. Although PMN interactions with the vessel walls were induced by inflammatory stimulus in all intestinal layers, TEM was restricted in the submucosa layer. $\mathbf{m}$ Time-lapse image sequence shows 2 PMNs exit the submucosa blood vessel at the same location over $5 \mathrm{~min}$ of image acquisition. Images were acquired at a rate of 10 frames per min and correspond to Video 5

respond differently during inflammation, specifically, to determine whether PMN TEM preferentially occurs in villus, submucosa, crypt or the muscularis layer.

Vasculature mapping in the intestinal layers by 3D confocal immunofluorescence microscopy revealed several distinct regions. The villi were (almost exclusively) comprised of an extensive network of small $(5-10 \mu \mathrm{m})$ vessels extending from each villus tip to its base. Each crypt base was encircled by a single vessel (5-10 $\mu \mathrm{m}$ in diameter). Just below this is the submucosal layer, which contained larger post-capillary venules (10-40 $\mu \mathrm{m}$ in diameter) fed by the crypt vessels. The larger submucosal venules also feed the relatively small $(5-10 \mu \mathrm{m})$ vessels of the outermost muscularis layer.

Real-time and time-lapse intravital imaging of the luminal surface (villi) in inflamed intestines revealed that despite the proximity to the intestinal epithelium, villus microvessels do not support PMN TEM. These findings are intriguing, as extravasation out of these vessels would constitute the shortest route for PMN 
a

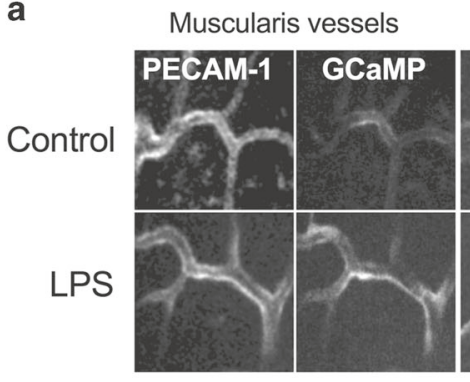

Submucosa vessels

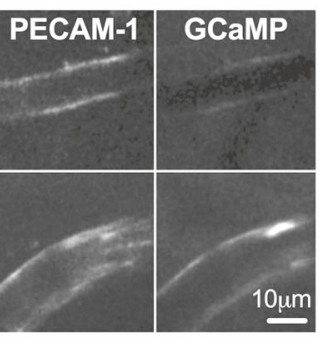

b

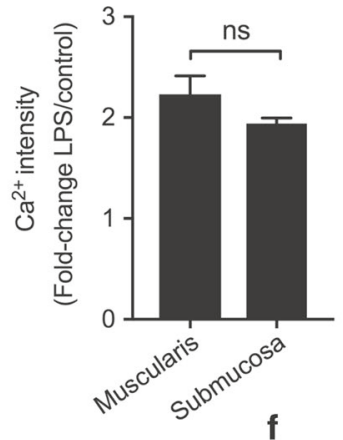

C

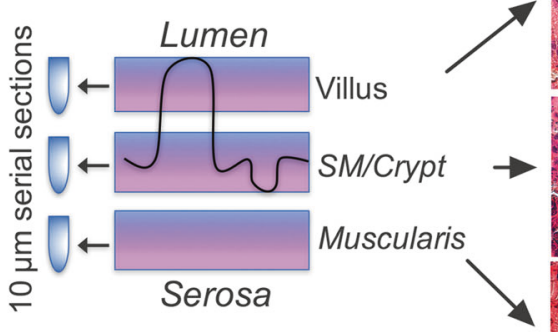

h

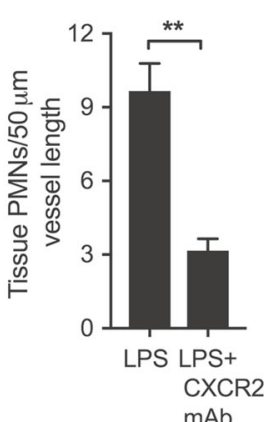

e

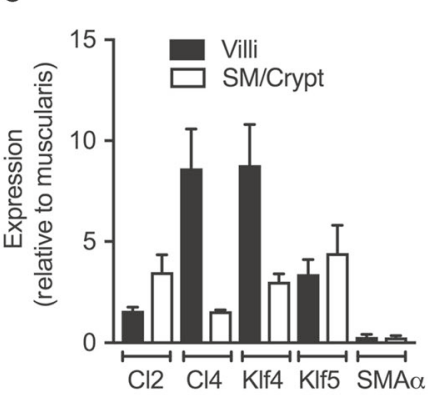

VIIII

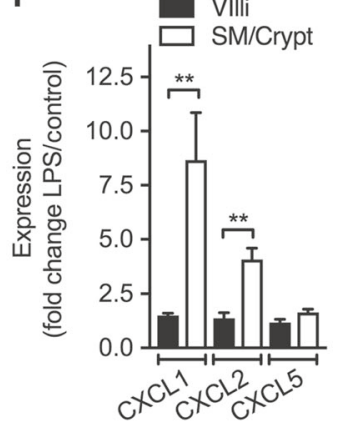

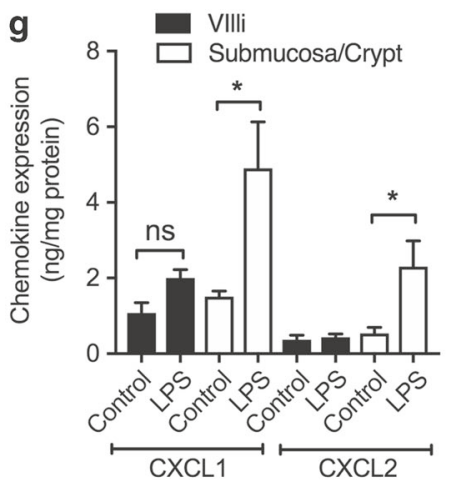

i

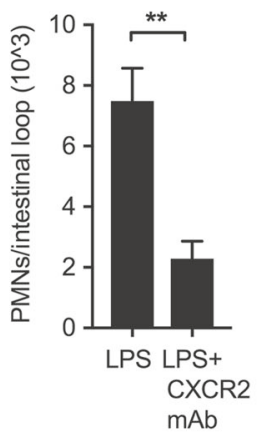

j

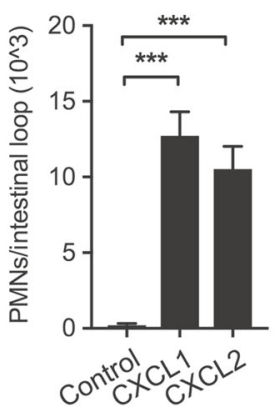

k

Fig. $6 \mathrm{CXCL1/CXCL2}$ and other intrinsic factors drive PMN TEM in the submucosa. a, b EC-specific Ca ${ }^{2+}$ reporter mice (VE-Cadherin-Cre GCaMP) were used in serosal imaging experiments to examine local changes in EC $\mathrm{Ca}^{2+}$ in the intestinal layers following stimulation with LPS. Vasculature was outlined by immunofluorescence staining for endothelial PECAM-1. a Representative images of at least four independent experiments and $\mathbf{b}$ quantification of fluorescence signal show no difference in the intracellular $\mathrm{Ca}^{2+}$ release by muscularis (left panels) and submucosa (right panels) vascular ECs following stimulation with LPS. Data are shown as an average of four independent experiments with at least 10 different fields quantitated per sample, ns not significant. c The schematic depicts intestinal layer separation by serial cryosectioning for expression and protein analysis. d Histological confirmation of layer separation by H\&E. e mRNA level of specific markers to each of the intestinal layers was determined by qRT-PCR to confirm separation. Data presented relative to the muscular layer and represent three independent experiments. The levels of CXCL1 and CXCL2 transcript (f) and protein (g) were examined using qRT-PCR and ELISA, respectively, and were found to be elevated in the submucosal/base crypt layer compared to villi following LPS stimulation. Data are shown as an average of four independent experiments, ns not significant, ${ }^{*} p<0.05,{ }^{* *} p<0.01$. $\mathbf{h}$ The number of tissue-infiltrated PMNs in the submucosa (quantified by IF in fixed tissue) and i PMN recruitment to the intestinal epithelium (analyzed by flow cytometry of digested tissue) in response to LPS-induced inflammation was significantly attenuated by Ab-mediated inhibition of CXCR2 (100 $\mu \mathrm{g} \mathrm{Ab}$ in $100 \mu \mathrm{l}$, i.p. injection). Data are shown as an average of three independent experiments, ${ }^{* *} p<0.01$. $\mathbf{~ P M N}$ recruitment to the intestinal epithelium in response to intraluminal administration of chemoattractants CXCL1 and CXCL2 $(2 \mathrm{~h})$ in ligated intestinal loop model was analyzed by flow cytometry. Data are shown as an average of five independent experiments, ${ }^{* * *} p<0.001$. k Real-time intravital imaging across the intestinal layers revealed that CXCL1and CXCL2-driven PMN TEM was restricted to the submucosa layer. Data are shown as an average of five independent experiments

migration toward the luminal space. Subsequent imaging from the serosal surface (muscularis, submucosa and crypt bases) revealed that PMN TEM was restricted to submucosal postcapillary venules. These intriguing observations suggest that PMN recruitment toward IECs and the luminal space, as seen in IBD or enteropathogenic infection, involves long-distance navigation of PMN through the interstitium, which may have significant implications on both PMN recruitment and function in inflamed intestines.
While PMN interstitial migration has not been examined in great detail, it has been suggested that this process is regulated by collagen density and the release of metalloproteinases. ${ }^{46}$ It can be further constrained by the extracellular matrix (ECM) structure and dependent upon actin-dependent shape change and 'squeezing' ability of migrating cells. ${ }^{47}$ Thus, PMN interstitial migration in inflamed intestinal mucosa may serve as an additional regulatory and limiting step for PMN influx into the intestinal tissue. Moreover, we have recently shown that 
actin-dependent PMN polarization leads to release of microvesicles containing effector proteins, including matrix metalloproteinases ${ }^{26}$ and chemokines, ${ }^{48}$ that can alter expression of key ECM proteins and facilitate recruitment of other immune cells. Ameboid migration of PMNs in 3D matrices and in vivo settings may further result in PMN degranulation ${ }^{49,50}$ and changes in their transcriptional profile, ${ }^{51,52}$ thus altering their functions upon arrival at the epithelial layers or the intestinal lumen. Indeed, emerging evidence demonstrates increased PMN plasticity, life span and phenotypic heterogeneity in inflamed tissue, ${ }^{53,54}$ which may explain their roles in both promoting tissue injury and facilitating resolution of inflammation.

Importantly, since PMNs can extravasate from small capillaries, as has been observed in the lung, ${ }^{19,20}$ our findings that PMN TEM was restricted to submucosal post-capillary venules suggest that blood vessels or the microenvironment in the intestinal layers may have different molecular or biophysical properties. Although the total $\mathrm{Ca}^{2+}$ response by ECs in the different strata was not different, we found elevated mRNA and protein levels of key PMN chemoattractants (CXCL1 and CXCL2) in the submucosa layer. Both CXCL1 and CXCL2 have known function in PMN recruitment; however, our work is the first to show their differential expression in the intestinal layers. Intriguingly, our findings suggest that although these chemokines contribute to PMN TEM in the submucosa, other crucial intrinsic differences in endothelial cells and the submucosa microenvironment also contribute to this process. We found that Ab-mediated inhibition of CXCR2 (a high-affinity receptor for both CXCL1 and CXCL2) resulted in significant reduction in PMN TEM; however, shifting the gradient of these chemokines (by luminal administration) from the submucosa to villus circulation was not sufficient to induce PMN TEM in these vessels.

As we further consider the physiological importance of restricted PMN TEM to the submucosa layer, it is tempting to speculate that the proximity of these vessels to several important immunological niches, including the crypt base, is not coincidental. PMNs that exit the submucosa blood vessels in these regions will likely encounter secretory cells, including Paneth and Goblet cells, intestinal stem cells and other resident interstitial cells. ${ }^{55}$ How PMNs may regulate tissue homeostasis through crosstalk with these local cells merits further investigation. It is also important to consider whether the observed extravasation patterns are unique to PMNs or also true for other cells that participate in gut immune responses, including monocytes, macrophages and T/B lymphocytes.

In summary, although PMN infiltration of the intestinal tissue is a well-known feature of gastrointestinal inflammation, how PMNs traffic across blood vessels and how they are recruited to inflamed epithelium are not known. Our findings define a distinct pattern of PMN recruitment in inflamed intestines and introduce submucosarestricted PMN TEM followed by interstitial migration as a new regulatory step in PMN trafficking. These unique patterns in the intestines provide targeting opportunities to limit PMN-mediated tissue injury in inflammatory disorders of the gastrointestinal tract.

\section{MATERIALS AND METHODS}

\section{Animals}

The following mouse strains aged 10-14 weeks were used in experimental protocols: C57BL6J mice (Jackson Laboratories), Ecadherin-mCFP mice (B6.129P2(Cg)-Cdh1tm1Cle, Jackson Laboratories) crossed with LysM-eGFP (Lyz2tm1.1Graf, gift from Dr. Perlman, Northwestern University) and bred to homozygosity, and VE-Cadherin (Cadherin5)-cre R26R mice ${ }^{56}$ crossed with GCaMP3 fl/ fl mice (a gift from Gangjian Qin, Northwestern University ${ }^{57}$ ) and bred to homozygosity. All mice were maintained under specific pathogen-free conditions at Northwestern University, Feinberg School of Medicine animal facilities. At the end of all experimental procedures animals were killed via rapid cervical dislocation. All protocols involving mice were reviewed and approved by the Institutional Animal Care and Use Committee at Northwestern University (PHS assurance number A328301).

\section{Antibodies and reagents}

Function-blocking CD11b (M1/70) and CXCR2 (242216) monoclonal antibodies were from eBioscience (San Diego, CA). An antiLy-6G and anti-CD11b conjugated to Alexa Fluor ${ }^{\circledR} 488$ were from Tonbo Bosciences (San Diego, CA). Non-blocking anti-PECAM-1 antibody (clone 390) was purchased from EMD Millipore (Burlington, MA) and conjugated to DyLight ${ }^{\circledR}-550$ using an antibody labeling kit from Thermo Fisher (Waltham, MA) according to the manufacture's instructions. PMN chemoattractants CXCL1 (KC) and CXCL2 (MIP2) were from Peprotec (Rocky Hill, NJ). Rat monoclonal E-cadherin (DECMA-1) was from ABCAM (Cambridge, MA). Phalloidin conjugated to Alexa Fluor ${ }^{\circledR} 647$ was from Invitrogen (Carlsbad, CA). Texas Red-labeled dextran (70 kDa) was from Molecular Probes (Eugene, OR). LPS, Hanks' balanced salt solution (HBSS) with $\mathrm{Ca} 2+$ and $\mathrm{Mg} 2+(\mathrm{HBSS}+)$ and HBSS without $\mathrm{Ca} 2+$ and $\mathrm{Mg} 2+(\mathrm{HBSS}-$ ) from Sigma (St Louis, MO).

\section{Inflammation models}

Salmonella infection. Mice were orally inoculated by gavage with streptomycin $(7.5 \mathrm{mg}$ in $200 \mu \mathrm{l}$ sterile water, $24 \mathrm{~h}$, renders mice more susceptible to oral Salmonella infection and intestinal colonization ${ }^{58,59}$ ) followed by $10^{8}$ Salmonella serovar typhimurium (in $200 \mu \mathrm{l}$ sterile PBS), leading to a gut-restricted inflammation. At $24 \mathrm{~h}$ following infection, mice were prepared for imaging experiments or killed and intestinal segments were excised for histological/flow cytometry examination.

Ligated intestinal loop model. Animals were anesthetized by subcutaneous intramascular injection of ketamine and xylazine mixture at doses of 100 and $5 \mathrm{mg} / \mathrm{kg}$, respectively. A midline abdominal incision was made and a $4 \mathrm{~cm}$ loop of small intestine was exteriorized and clipped at proximal and distal ends. After luminal administration of LPS $(100 \mu \mathrm{g} / \mathrm{ml}$ in $200 \mu \mathrm{l} \mathrm{HBSS}+)$ or PMN chemoattractants CXCL1 (KC) or CXCL2 (MIP2) $(1 \mu \mathrm{M}$ in $200 \mu \mathrm{l}$ $\mathrm{HBSS}+$ ), excised loops were reinserted into the peritoneal cavity for $1-2 \mathrm{~h}$ of incubation period. ${ }^{26,45}$ Where indicated, functionblocking anti-CXCR2 antibody ( $100 \mu \mathrm{g}$ in $100 \mu \mathrm{l})$ was administered i.p. 40 min prior to LPS administration, and anti-CD11b or antiICAM-1 Abs $(50 \mu \mathrm{g} / \mathrm{ml})$ were administered via i.v. injection prior to the induction of inflammation.

Intravital imaging

Animal setup. Subsequent to induction of inflammation (described above), mice were anesthetized, and a small vertical incision was made to expose small intestinal segments. Without further perturbing the tissue, mice were placed on a heated platform $\left(37^{\circ} \mathrm{C}\right)$ and control or inflamed intestinal segments were secured onto a custom-made chamber that was developed and elegantly described in McDole et al., ${ }^{28}$ with minor modifications, as detailed below. This setup allows imaging with minimal tissue manipulation. For imaging experiments, a non-blocking antiPECAM-1 Ab conjugated to DyLight ${ }^{\oplus}-550$ was administered i.v. (retro-orbital) $30 \mathrm{~min}$ prior to imaging to visualize blood vessels.

For serosal imaging, a segment of the small intestine was secured by Vetbond tissue adhesive (3M) between two glass coverslips, one attached to the bottom of the upper chamber plate, located over the incision in the mouse's abdomen, and second a smaller one placed under the intestinal segment and reinserted into the peritoneal cavity. Securing the tissue in such way was sufficient in most cases to overcome peristaltic movement of the small intestine without additional treatments. The imaging chamber was heated and maintained at $37^{\circ} \mathrm{C}$ and tissue was hydrated by the addition of a small amount of phenol red-free RPMI- 1640 at $37^{\circ} \mathrm{C}$ as needed. 
For imaging from the luminal surface (villi) an additional incision was made along the dorsal side of the intestinal segment to expose the epithelium lining the intestinal lumen. Care was taken to avoid large blood vessels. The intestine was then washed several times to remove fecal material and mucus from luminal surface and bathed in phenol red-free RPMI- 1640 at $37^{\circ} \mathrm{C}$. In select experiments, the intestinal lumen was outlined by an intraluminal injection of Dextran-Texas Red MW $70 \mathrm{kDa}(10 \mu \mathrm{g} / \mathrm{ml}$, $20 \mathrm{~min}$ prior to imaging). Molecular weight (MW) $70 \mathrm{kDa}$ dextran was used as lower MW dextrans (i.e., $10 \mathrm{kDa}$ or $40 \mathrm{kDa}$ ) leak into the interstitium upon induction of inflammation and alteration in the epithelial integrity. ${ }^{60}$ All imaging experiments were performed by using either multiphoton or spinning disk confocal microscopy.

Multiphoton imaging. Where indicated, the Nikon A1R-MP upright multiphoton microscope was used for analysis of PMN rolling/adhesion interactions at $30 \mathrm{fps}$ acquisition speed with frame averaging for clarity $(\times 2)$, and the $x-y$ dimensions of the scan area set at $512 \times 512 \mu \mathrm{m}$. The microscope was equipped with a Coherent (Santa Clara, CA) tunable titanium-sapphire Chameleon laser system tuned to: $830 \mathrm{~nm}$ for the CFP/GFP analysis, 900 $\mathrm{nm}$ to visualize Texas Red and $900 \mathrm{~nm}$ for Alexa Fluor ${ }^{\circledR}$ 647. For $Z$ stacks of live or fixed tissue, images were acquired sequentially to $80 z$-steps ( $2.0 \mu \mathrm{m}$ each). Time-lapse imaging was performed at 10 fpm.

Spinning disk confocal imaging. The UltraVIEW VoX imaging system was built on an Olympus BX-51WI Fixed Stage illuminator and equipped with a Yokogawa CSU-X1-A1 spinning disk, a Hamamatsu EMCCD C9100-50 camera and a Modular Laser System with solid state diode lasers with DPPS modules for 488 , 561 , and $640 \mathrm{~nm}$ and the appropriate filters (all assembled by Perkin Elmer, Naperville, IL). Synchronization was managed by a Prosync 2 Syncronization Controller. Z-axis movement and objective positioning was controlled by Piezoelectric MIPOS100 System (Piezoystem Jena, Germany). Images were collected using a $20 \times$ water-immersion objective (1.00 numerical aperture). Volocity ${ }^{\circledR}$ software (Perkin Elmer) was used to drive the microscopy and acquire images, which were then analyzed using ImageJ. LysM-eGFP is a well-accepted model for studying PMN trafficking. However, because LysM-eGFP is also expressed by tissue macrophages and monocytes, control experiments were performed with additional labeling of Ly6G (i.v.) to specifically identify PMNs. As expected in an acute inflammatory setting, where PMNs are the first responders, the majority $(>90 \%)$ of cells in the tissue were PMNs (Supplementary Fig $1 \mathrm{~A})$. Rare $(<5 \%)$, LysM-eGFP-positive, Ly6G-negative monocyte/inflammatory macrophages were seen (Supplementary Fig 1B). Finally, as shown in Supplementary Fig 1C, eGFP ${ }^{\text {hi }}$ tissue PMNs could be clearly distinguished from eGFPlow tissue macrophages (by both morphology and GFP intensity, such that monocyte/macrophage signal did not interfere with tissue PMN analyses). Data analysis was performed using ImageJ and Imaris software.

For assessment of $\mathrm{EC} \mathrm{Ca}{ }^{2+}$ responses, EC-specific $\mathrm{Ca}^{2+}$ reporter mice (VE-Cadherin-cre GCaMP) were used in serosal imaging experiments and imaged using the spinning disk microscope. Fluorescence intensity as an indicator of intracellular $\mathrm{Ca}^{2+}$ level was quantified in separate experiments with and without LPS stimulation (administered intraluminally into ligated intestinal loops for $1 \mathrm{~h}$ ).

Analyses of PMN-EC interactions. Cell flux was defined as the number of cells that were visualized in the field of view, per $30 \mathrm{~s}$ acquisition time. Free-flowing cells were defined as cells recorded passing through a blood vessel without interacting with the vessel wall. Rolling cells were defined as cells that have remained in continued contact with the vessel wall for greater than $5 \mathrm{~s}$, otherwise cells were defined as tethered. ${ }^{61}$ Adherent cells were defined as cells that remained attached to the vessel wall for $>20$ s.

Immunofluorescence labeling

Cryosections ( $8 \mu \mathrm{m}$ width) of O.C.T. (optimal cutting temperature)-frozen intestinal tissue were ethanol fixed, blocked with $5 \%$ bovine serum albumin in PBS and incubated with the relevant primary $\mathrm{Ab}\left(10 \mu \mathrm{g} / \mathrm{ml}\right.$, overnight at $\left.4{ }^{\circ} \mathrm{C}\right)$ either directly conjugated or followed by an appropriate fluorescently labeled secondary antibody ( $1 \mathrm{~h}$ at room temperature). Alternatively, whole mount tissue staining was performed by isolating a small segment of small intestine, pinning it flat onto a silicon-coated Petri-dish and immunostaining as above. All images were captured using a Nikon A1R+ confocal microscope with $60 \times$ oil objective.

\section{Flow cytometry}

Following the relevant treatment, intestinal loops were longitudinally opened. The epithelium and the PMNs that were associated with the epithelial layer (IEC-associated) were dissociated from the rest of the tissue by fragmentation and shaking in $2 \mathrm{mM}$ dithiothreitol (DTT) and $5 \mathrm{mM}$ EDTA in HBSS+ solution $(3-5 \times 10$ min shakes, until no more epithelial cells were visible in the solution as previously described $\left.{ }^{23,24}\right)$. Only a small number of macrophages ( $<3 \%$ of all $C D 45^{+}$immune cell), which typically reside within the $L P,{ }^{24}$ were detected in this fraction, suggesting minimal contamination by LP immune cells (not shown). Where specified, PMNs were further extracted from the LP of control and inflamed intestinal segments using a collagenase-mediated tissue digestion as previously described, ${ }^{25}$ which separates the LP from the underlying muscularis layer. The number of CD $45^{+}$CD $11 b^{+}{\text {Ly } 6 G^{+}}$PMNs in each fraction was quantified using BD FACS CANTO II and FlowJo software.

\section{Separation of intestinal layers}

Intestinal layers were separated as previously described. ${ }^{42}$ Briefly, a segment of a small intestine $(\sim 0.5 \times 1 \mathrm{~cm})$ was longitudinally opened, laid flat between two metal plates and fixed in O.C.T. Intestinal tissue was then sectioned en face along the long axis with section width set to $10 \mu \mathrm{m}$. Five sections were recovered for each layer: luminal surface (villi), submucosa (submucosa/crypts) and serosal surface (muscularis). Three transitional sections between the layers were discarded. Sections for each layer were pooled and processed for mRNA and protein analysis. Separation of intestinal layers was confirmed visually using light microscopy, by hematoxylin and eosin (H\&E) staining and by expression of specific markers.

ELISA

CXCL1 and CXCL2 were quantitated from sectioned intestinal layers using standard sandwich ELISA methods. Briefly, tissue sections were lysed in Dulbecco's PBS supplemented with $1.0 \%$ Nonidet P-40, $0.1 \%$ sodium dodecyl sulfate, $1 \times$ protease inhibitor cocktail and $0.5 \mathrm{mM}$ phenylmethylsulfonyl fluoride. Samples were homogenized by 20 passes through a $21 \mathrm{G}$ needle and cleared of insoluble material by centrifugation at $18,000 \times g$ at $4{ }^{\circ} \mathrm{C}$ for $15 \mathrm{~min}$. Cleared homogenate was tested for the abundance of CXCL1 and CXCL2 using sandwich ELISA kits from LifeSpan BioSciences, Inc. (LS-F31327 and LS-F31334 respectively). Samples were compared to standard curves that were included in each assay and normalized to protein concentrations determined using a standard micro BCA assay (Thermo Fisher). No cross interference between CXCL1 and CXCL2 was detected, suggesting the reagents were highly specific for their intended targets. Also, the effect of small amounts of O.C.T. was confirmed to not interfere with both ELISAs and the micro BCA protein determination. 


\section{Statistics}

Statistical significance was assessed by Student's $t$-test or by oneway analysis of variance with Newman-Keuls multiple comparison test using Graphpad Prism (V4.0). Statistical significance was set at $p<0.05$. For all experiments the data shown as \pm SEM.

\section{ACKNOWLEDGEMENTS}

This work was supported by grants from the National Institutes of Health (NIH) DK101675, Digestive Health Foundation and by the Robert H. Lurie Comprehensive Cancer Center to R.S. and NIH R01 HL046849 and R37 HL064774 to W.A.M. We thank the Northwestern University Center for Advanced Microscopy core (supported by NCl CCSG P30 CA060553 awarded to the Robert H. Lurie Comprehensive Cancer Center) for the help with the imaging experiments. Multiphoton microscopy was performed on a Nikon A1R multiphoton microscope, acquired through the support of $\mathrm{NIH}$ 1S10OD010398-01.

\section{AUTHOR CONTRIBUTIONS}

D.P.S, T.B., V.B.-I. and R.S. performed experiments and analyzed results; R.S. wrote the manuscript; all authors edited and revised the manuscript.

\section{ADDITIONAL INFORMATION}

The online version of this article (https://doi.org/10.1038/s41385-018-0069-5) contains supplementary material, which is available to authorized users.

Competing interests: The authors declare no competing interests.

\section{REFERENCES}

1. Parkos, C. A. Neutrophil-epithelial interactions: a double-edged sword. Am. J. Pathol. 186, 1404-1416 (2016).

2. Xavier, R. J. \& Podolsky, D. K. Unravelling the pathogenesis of inflammatory bowel disease. Nature 448, 427-434 (2007).

3. Ma, T. Y. Intestinal epithelial barrier dysfunction in Crohn's disease. Proc. Soc. Exp. Biol. Med. 214, 318-327 (1997).

4. Nusrat, A., Parkos, C. A., Liang, T. W., Carnes, D. K. \& Madara, J. L. Neutrophil migration across model intestinal epithelia: monolayer disruption and subsequent events in epithelial repair. Gastroenterology 113, 1489-1500 (1997).

5. Toumi, H., F'Guyer, S. \& Best, T. M. The role of neutrophils in injury and repair following muscle stretch. J. Anat. 208, 459-470 (2006).

6. Zemans, R. L. et al. Neutrophil transmigration triggers repair of the lung epithelium via beta-catenin signaling. Proc. Natl. Acad. Sci. USA 108, 15990-15995 (2011).

7. Borgstein, R. L., Shaw, J. J. \& Pearson, R. H. Uterine leiomyomata: sonographic mimicry. Br. J. Radiol. 62, 1019-1021 (1989).

8. Fournier, B. M. \& Parkos, C. A. The role of neutrophils during intestinal inflammation. Mucosal Immunol. 5, 354-366 (2012).

9. Sumagin, R. et al. Neutrophil interactions with epithelial-expressed ICAM-1 enhances intestinal mucosal wound healing. Mucosal Immunol. 9, 1151-1162 (2016).

10. Baumler, A. J. \& Sperandio, V. Interactions between the microbiota and pathogenic bacteria in the gut. Nature 535, 85-93 (2016).

11. Hickey, M. J. \& Westhorpe, C. L. Imaging inflammatory leukocyte recruitment in kidney, lung and liver--challenges to the multi-step paradigm. Immunol. Cell Biol. 91, 281-289 (2013).

12. Sarafidis, K. et al. Evidence of early systemic activation and transendothelial migration of neutrophils in neonates with severe respiratory distress syndrome. Pediatr. Pulmonol. 31, 214-219 (2001).

13. Sumagin, R. \& Sarelius, I. H. Intercellular adhesion molecule-1 enrichment near tricellular endothelial junctions is preferentially associated with leukocyte transmigration and signals for reorganization of these junctions to accommodate leukocyte passage. J. Immunol. 184, 5242-5252 (2010).

14. Ortolan, E. et al. CD157 plays a pivotal role in neutrophil transendothelial migration. Blood 108, 4214-4222 (2006).

15. Sumagin, R., Lomakina, E. \& Sarelius, I. H. Leukocyte-endothelial cell interactions are linked to vascular permeability via ICAM-1-mediated signaling. Am. J. Physiol. Heart Circ. Physiol. 295, H969-H977 (2008).

16. Bathe, M., Shirai, A., Doerschuk, C. M. \& Kamm, R. D. Neutrophil transit times through pulmonary capillaries: the effects of capillary geometry and fMLPstimulation. Biophys. J. 83, 1917-1933 (2002).

17. Muller, W. A. Mechanisms of leukocyte transendothelial migration. Annu. Rev. Pathol. 6, 323-344 (2011).
18. Ley, K., Laudanna, C., Cybulsky, M. I. \& Nourshargh, S. Getting to the site of inflammation: the leukocyte adhesion cascade updated. Nat. Rev. Immunol. 7, 678-689 (2007).

19. Burns, A. R., Smith, C. W. \& Walker, D. C. Unique structural features that influence neutrophil emigration into the lung. Physiol. Rev. 83, 309-336 (2003).

20. Doerschuk, C. M. Mechanisms of leukocyte sequestration in inflamed lungs. Microcirculation 8, 71-88 (2001).

21. Nourshargh, S. \& Alon, R. Leukocyte migration into inflamed tissues. Immunity $\mathbf{4 1}$, 694-707 (2014).

22. Criss, A. K., Silva, M., Casanova, J. E. \& McCormick, B. A. Regulation of Salmonellainduced neutrophil transmigration by epithelial ADP-ribosylation factor 6. J. Biol. Chem. 276, 48431-48439 (2001).

23. Davies, M. D. \& Parrott, D. M. Preparation and purification of lymphocytes from the epithelium and lamina propria of murine small intestine. Gut 22, 481-488 (1981).

24. Goodyear, A. W., Kumar, A., Dow, S. \& Ryan, E. P. Optimization of murine small intestine leukocyte isolation for global immune phenotype analysis. J. Immunol. Methods 405, 97-108 (2014).

25. Weigmann, B. et al. Isolation and subsequent analysis of murine lamina propria mononuclear cells from colonic tissue. Nat. Protoc. 2, 2307-2311 (2007).

26. Butin-Israeli, V. et al. Deposition of microparticles by neutrophils onto inflamed epithelium: a new mechanism to disrupt epithelial intercellular adhesions and promote transepithelial migration. FASEB J. 30, 4007-4020 (2016).

27. Mizuno, R. et al. In vivo imaging reveals PKA regulation of ERK activity during neutrophil recruitment to inflamed intestines. J. Exp. Med. 211, 1123-1136 (2014).

28. McDole, J. R. et al. Goblet cells deliver luminal antigen to CD103+dendritic cells in the small intestine. Nature 483, 345-349 (2012).

29. Chieppa, M., Rescigno, M., Huang, A. Y. \& Germain, R. N. Dynamic imaging of dendritic cell extension into the small bowel lumen in response to epithelial cell TLR engagement. J. Exp. Med. 203, 2841-2852 (2006).

30. Benjamim, C. F. et al. Inhibition of leukocyte rolling by nitric oxide during sepsis leads to reduced migration of active microbicidal neutrophils. Infect. Immun. 70, 3602-3610 (2002).

31. Allingham, M. J., van Buul, J. D. \& Burridge, K. ICAM-1-mediated, Src- and Pyk2dependent vascular endothelial cadherin tyrosine phosphorylation is required for leukocyte transendothelial migration. J. Immunol. 179, 4053-4064 (2007).

32. Sumagin, R., Prizant, H., Lomakina, E., Waugh, R. E. \& Sarelius, I. H. LFA-1 and Mac1 define characteristically different intralumenal crawling and emigration patterns for monocytes and neutrophils in situ. J. Immunol. 185, 7057-7066 (2010).

33. Sullivan, D. P., Watson, R. L. \& Muller, W. A. 4D intravital microscopy uncovers critical strain differences for the roles of PECAM and CD99 in leukocyte diapedesis. Am. J. Physiol. Heart Circ. Physiol. 311, H621-H632 (2016).

34. Woodfin, A. et al. The junctional adhesion molecule JAM-C regulates polarized transendothelial migration of neutrophils in vivo. Nat. Immunol. 12, 761-769 (2011).

35. Taniguchi, T. et al. Plasmodium berghei ANKA causes intestinal malaria associated with dysbiosis. Sci. Rep. 5, 15699 (2015).

36. Mowat, A. M. \& Agace, W. W. Regional specialization within the intestinal immune system. Nat. Rev. Immunol. 14, 667-685 (2014).

37. Huang, A. J. et al. Endothelial cell cytosolic free calcium regulates neutrophil migration across monolayers of endothelial cells. J. Cell Biol. 120, 1371-1380 (1993).

38. Weber, E. W. et al. TRPC6 is the endothelial calcium channel that regulates leukocyte transendothelial migration during the inflammatory response. J. Exp. Med. 212, 1883-1899 (2015).

39. Zariwala, H. A. et al. A Cre-dependent GCaMP3 reporter mouse for neuronal imaging in vivo. J. Neurosci. 32, 3131-3141 (2012).

40. Kolaczkowska, E. \& Kubes, P. Neutrophil recruitment and function in health and inflammation. Nat. Rev. Immunol. 13, 159-175 (2013).

41. Williams, M. R., Azcutia, V., Newton, G., Alcaide, P. \& Luscinskas, F. W. Emerging mechanisms of neutrophil recruitment across endothelium. Trends Immunol. 32, 461-469 (2011).

42. Farkas, A. E., Gerner-Smidt, C., Lili, L., Nusrat, A. \& Capaldo, C. T. Cryosectioning method for microdissection of murine colonic mucosa.J. Vis. Exp. 101, e53112 (2015).

43. Ajuebor, M. N. \& Swain, M. G. Role of chemokines and chemokine receptors in the gastrointestinal tract. Immunology 105, 137-143 (2002).

44. Belperio, J. A. et al. Critical role for CXCR2 and CXCR2 ligands during the pathogenesis of ventilator-induced lung injury. J. Clin. Invest. 110, 1703-1716 (2002).

45. Sumagin, R., Robin, A. Z., Nusrat, A. \& Parkos, C. A. Transmigrated neutrophils in the intestinal lumen engage ICAM-1 to regulate the epithelial barrier and neutrophil recruitment. Mucosal Immunol. 7, 905-915 (2014). 
46. Lerchenberger, M. et al. Matrix metalloproteinases modulate ameboid-like migration of neutrophils through inflamed interstitial tissue. Blood 122 770-780 (2013).

47. Lammermann, T. et al. Rapid leukocyte migration by integrin-independent flowing and squeezing. Nature 453, 51-55 (2008).

48. Lim, K. et al. Neutrophil trails guide influenza-specific CD8(+) T cells in the airways. Science 349, aaa4352 (2015).

49. Brazil, J. C., Sumagin, R., Cummings, R. D., Louis, N. A. \& Parkos, C. A. Targeting of neutrophil Lewis $X$ blocks transepithelial migration and increases phagocytosis and degranulation. Am. J. Pathol. 186, 297-311 (2016).

50. Itou, T., Collins, L. V., Thoren, F. B., Dahlgren, C. \& Karlsson, A. Changes in activation states of murine polymorphonuclear leukocytes (PMN) during inflammation: a comparison of bone marrow and peritoneal exudate PMN. Clin. Vaccin. Immunol. 13, 575-583 (2006).

51. Tsukahara, Y. et al. Gene expression in human neutrophils during activation and priming by bacterial lipopolysaccharide. J. Cell. Biochem. 89, 848-861 (2003).

52. Martinez, F. O. et al. IL-8 induces a specific transcriptional profile in human neutrophils: synergism with LPS for IL-1 production. Eur. J. Immunol. 34, 2286-2292 (2004).

53. Sagiv, J. Y. et al. Phenotypic diversity and plasticity in circulating neutrophil subpopulations in cancer. Cell Rep. 10, 562-573 (2015).
54. Perobelli, S. M. et al. Plasticity of neutrophils reveals modulatory capacity. Braz. J. Med. Biol. Res. 48, 665-675 (2015).

55. Clevers, H. The intestinal crypt, a prototype stem cell compartment. Cell 154 274-284 (2013).

56. Alva, J. A. et al. VE-Cadherin-Cre-recombinase transgenic mouse: a tool for lineage analysis and gene deletion in endothelial cells. Dev. Dyn. 235, 759-767 (2006).

57. Tian, L. et al. Imaging neural activity in worms, flies and mice with improved GCaMP calcium indicators. Nat. Methods 6, 875-881 (2009).

58. Barthel, M. et al. Pretreatment of mice with streptomycin provides a Salmonella enterica serovar Typhimurium colitis model that allows analysis of both pathogen and host. Infect. Immun. 71, 2839-2858 (2003).

59. Hapfelmeier, S. \& Hardt, W. D. A mouse model for S. typhimurium-induced enterocolitis. Trends Microbiol. 13, 497-503 (2005).

60. Monteiro, A. C. et al. JAM-A associates with ZO-2, afadin, and PDZ-GEF1 to activate Rap2c and regulate epithelial barrier function. Mol. Biol. Cell 24, 2849-2860 (2013).

61. Lawrence, M. B., Bainton, D. F. \& Springer, T. A. Neutrophil tethering to and rolling on E-selectin are separable by requirement for L-selectin. Immunity 1, 137-145 (1994). 